\title{
CARACTERIZACIÓN DEL ESPECTRO DISDROMÉTRICO DE LA PRECIPITACIÓN Y APLICACIONES
}

\author{
Marta ANGULO-MARTÍNEZ1 ${ }^{1}$, Santiago BEGUERÍA ${ }^{1}$ \\ ${ }^{1}$ Estación Experimental de Aula Dei, Consejo Superior de Investigaciones Cientificas (EEAD-CSIC), \\ Zaragoza. \\ marta.angulo@eead.csic, santiago.begueria@csic.es
}

\section{RESUMEN}

Los nuevos sistemas de medida basados en el carácter intrínsecamente discreto de la precipitación (corriente de partículas individuales: gotas de lluvia, copos de nieve, etc.) han incrementado la precisión en el registro de eventos de precipitación. Estos instrumentos de medida, denominados disdrómetros ópticos o espectropluviómetros, son capaces de caracterizar el espectro disdrométrico de la precipitación o distribución de frecuencias del tamaño y velocidad de las gotas. Esta información permite calcular variables integradas como la densidad de gotas o la masa de agua por unidad de volumen de aire, la intensidad y volumen de la precipitación, la energía cinética, la visibilidad o la reflectividad de radar, además de clasificar los meteoros (lluvia, lluvia fina, granizo, nieve, etc). Más allá del interés aplicado de estas variables, el análisis de la información disdrométrica durante episodios de precipitación concretos permite indagar sobre los mecanismos genéticos y de evolución de los meteoros. La información disdrométrica junto con el radar meteorológico permite validar la precipitación obtenida a través de los satélites u otros medios de teledetección de la precipitación. En esta comunicación se presenta una revisión de la información disdrométrica y su evolución, con ejemplos de aplicación.

Palabras clave: disdrómetro; dsd; radar meteorológico; teledetección de la precipitación.

\begin{abstract}
New precipitation measurement devices, based on the precipitation discrete character (individual particles flow: as raindrops, snowflakes, etc.) have improved the accuracy in recording precipitation events and its characteristics. These devices, known as optical disdrometers or spectropluviometers allow characterizing the precipitation particle spectrum or the size and velocity frequency distribution of the precipitation particles. This information allows estimating integrate values as raindrops density, the water content per volume of air, precipitation intensity and precipitation amount, kinetic energy, visibility and radar reflectivity, besides hydrometeors classification (rain, drizzle, hail, snow, etc). Apart from the applied interest, the analysis of precipitation particle spectrum, during precipitation events, informs on precipitation genetic mechanisms and their evolution. In addition, disdrometers, in combination with meteorological radars, are useful tools for validating precipitation satellite data, and other remote sensing precipitation products. This article reviews the measurement evolution of disdrometric information providing some application examples.
\end{abstract}


Key words: disdrometer; dsd; weather radar; remote sensing.

\section{EVOLUCIÓN DEL ESTUDIO SOBRE EL ESPECTRO DISDROMÉTRICO DE LA PRECIPITACIÓN}

El interés por determinar la distribución de los tamaños de las gotas de lluvia asociado a los distintos tipos de precipitación surgió alrededor de finales-principios de siglo XIX-XX, con el objetivo de conocer, entender y caracterizar más en profundidad la precipitación, desde un punto de vista meteorológico. Destacan en este sentido, los estudios seminales de Lowe (1892) y Bentley (1904), quienes estudiaron el espectro disdrométrico de la precipitación a partir de los datos obtenidos por la impresión de las gotas de lluvia sobre papel tintado. En efecto, los primeros métodos utilizados para conocer el espectro disdrométrico de la precipitación y su relación con los distintos tipos e hidrometeoros, eran métodos tediosos y con gran incertidumbre, que necesitaban un gran esfuerzo humano pues carecían de automatización. Entre estos, destacan: el método del papel tintado o papel de filtro teñido en el que las gotas dejaban una huella de su forma al chocar contra el papel (filter paper), existiendo una relación previamente calibrada, entre el tamaño de la huella y el de la gota. Otro método similar era el de las bolitas de harina (flour pellet), que aprovechaba la capacidad de amalgamación de las gotas al contacto con la harina, resultando en gotas de tamaño equivalente que, una vez secas, eran medidas.

Esta información ya permitió empezar a conocer que existían diferencias entre tipos de lluvia y la forma en la que las gotas asociadas se distribuían. En especial, comenzó a observarse que la distribución de las gotas variaba con la intensidad de la precipitación (Laws and Parsons, 1943), y ésta dependía del tipo de evento, estratiforme vs. convectivo, y de características geográficas como la elevación y la proximidad al mar, entre otras (Blanchard, 1953).

Inicialmente, uno de los ámbitos científicos donde este tipo de información era de gran interés aplicado fue la agricultura, especialmente se trataba de investigar las causas de erosión de suelo; tanto la erosión por la salpicadura de las gotas en el suelo, como la asociada a la producción por escorrentía. Por ello, este campo científico y aplicado ha sido muy prolífico en las publicaciones relacionadas con el espectro disdrométrico de la precipitación y desarrollos instrumentales para su mejor caracterización (Fig. 1). En este ámbito científico, una de las finalidades fundamentales es la de encontrar una buena relación entre la energía cinética de la precipitación y la intensidad, con el fin de determinar el potencial erosivo de la precipitación en modelos espacialmente distribuidos, como la Ecuación Universal de Erosión de Suelo (RUSLE, Renard et al., 1997).

Las mediciones disdrométricas basadas en métodos manuales, como los mencionados, no permitían muestreos continuos automatizados. A finales de la década de los 60s y principios de los 70s, comenzaron a aplicarse los primeros disdrómetros. Éstos estaban basados bien en fotografías o videos de alta frecuencia (Mutchler y Hansen, 1970) o en la detección de la onda de presión generada por el impacto de las gotas de lluvia sobre una superficie (Joss y Waldvogel, 1967), también conocido como disdrómetro acústico. Ambas tipologías se emplearon tanto en estudios relacionados con la erosión del suelo como en otras disciplinas, más relacionadas con el radar meteorológico y la teledetección de la precipitación. 


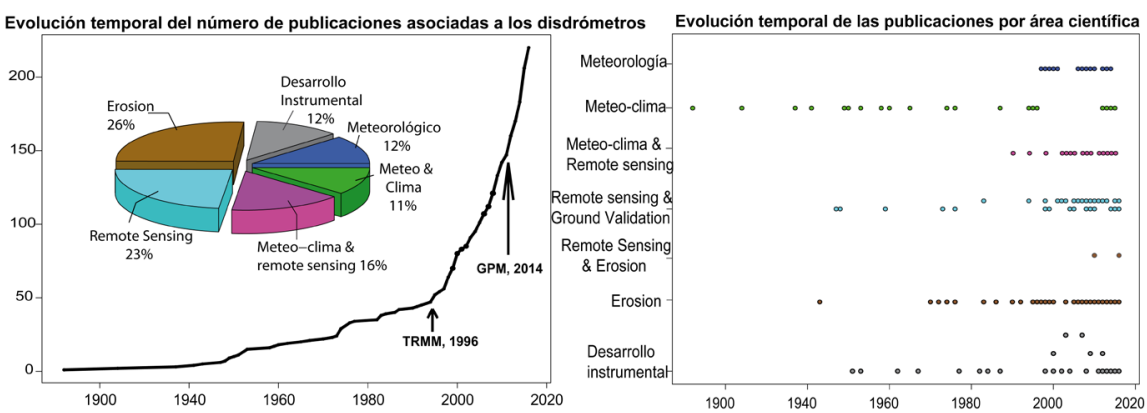

Fig. 1: Evolución temporal del número de publicaciones por áreas científicas asociado al espectro disdrométrico de la precipitación. Fuente: elaboración propia.

Durante la II Guerra Mundial, los operadores de radar notaban ruido en ecos de retorno debido a elementos meteorológicos. Una vez acabada ésta surgió una nueva línea de investigación enfocada al estudio de la precipitación mediante tecnología radar. El radar es una medida indirecta. Los hidrometeoros suspendidos en la atmósfera se detectan a través de la reflectividad de la señal (Z). En este sentido, la determinación de la forma, el tamaño y la velocidad de los meteoros es necesaria para poder estimar correctamente la relación entre la reflectividad $(Z)$ y la intensidad de la precipitación (R) y las posibles aplicaciones de estas medidas. Los disdrómetros permiten una mejor estimación de la relación Z-R, ya que proporcionan medidas directas de las características discretas de los hidrometeoros, de las que se derivan medidas integradas como la intensidad, o el volumen de agua.
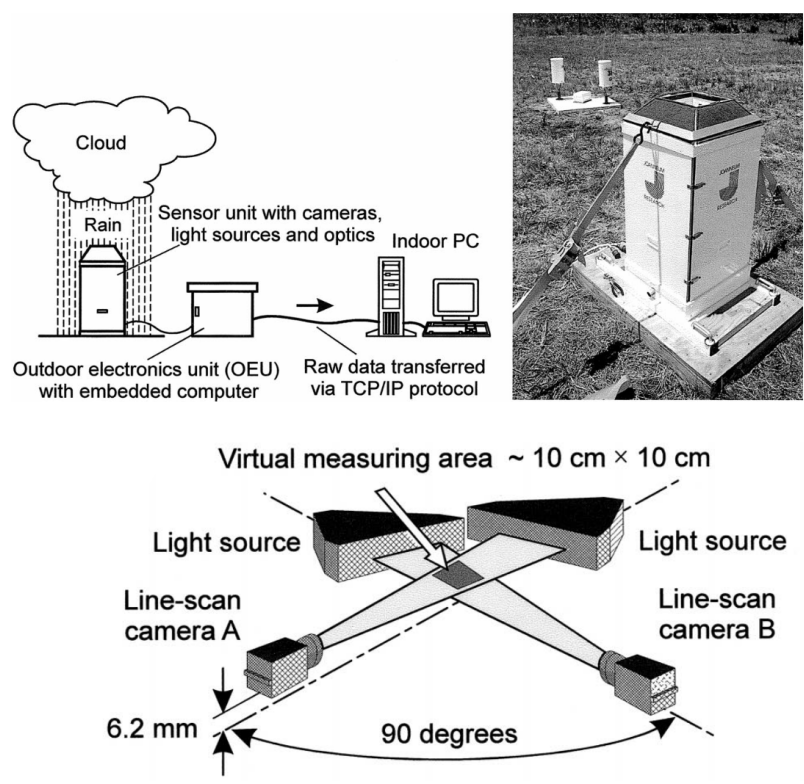

Fig. 2: Video disdrómetro de dos dimensiones, (2DVD). Fuente: Kruger y Krajewski, 2002. 
Por ello, los avances científicos en el radar meteorológico u otras formas de teledetección de la precipitación han impulsado también el desarrollo instrumental de aparatos capaces de medir con exactitud el espectro disdrométrico. Asimismo, y basado en la tecnología láser, surge el disdrómetro óptico (Hauser et al., 1984) que se ha ido perfeccionando hasta los actuales, como OTT Parsivel o Thies Clima Laser Precipitation Monitor, siendo además relativamente asequibles.

Además de la diferencia en ambas medidas, resulta importante comentar que los disdrómetros ofrecen medidas puntuales, mientras que el radar aporta una información tridimensional, lo cual hace necesaria la aplicación de modelos capaces de contrarrestar tales diferencias. Asimismo, los disdrómetros no están exentos de error y para poder ofrecer medidas precisas de la forma, tamaño y velocidad de los hidrometeoros han de ser capaces de medir tridimensionalmente. Actualmente, el video disdrómetro de dos dimensiones (2DVD; Kruger y Krajewski, 2002) está diseñado con ese fin, (Fig. 2).

\section{APLICACIONES DE LA INFORMACIÓN DISDROMÉTRICA DE LA PRECIPITACIÓN}

El espectro disdrométrico de la precipitación es la información más completa sobre la precipitación que se puede analizar, por ello es muy importante y transversal para varios campos científicos así como para aplicaciones comerciales e industriales. Algunos ejemplos incluyen: la teledetección de la precipitación, la meteorología y climatología, los modelos climáticos, modelos numéricos y de incertidumbre aplicados al radar meteorológico y/o la detección de la precipitación por satélite, así como aplicaciones dentro del ámbito agronómico y de erosión y conservación de los usos del suelo; en última instancia, para una buena determinación de los balances hídricos y los riesgos climáticos en relación con los ecosistemas y la vida de las personas.

La figura 1 mostraba claramente la importancia de dos ámbitos de estudio sobre los restantes: la erosión del suelo y la teledetección de la precipitación.

En el ámbito de la erosión del suelo resulta necesario determinar la capacidad de la precipitación para desagregar y movilizar partículas de suelo, a través de la energía cinética de las gotas. En este aspecto se unen los campos meteorológico, climatológico, edafológico, agronómico y ambiental.

Tal y como se ha comentado, muchos de los estudios en este apartado se han enfocado en buscar relaciones entre la energía cinética de la precipitación y la intensidad, con el fin de poder incluir el carácter discreto-la fuerza de las gotas, al continuo, es decir, el volumen de agua precipitado por unidad de tiempo. Como esta última variable es de fácil obtención y está disponible con una buena cobertura espacial en muchos lugares de la Tierra, resulta necesario encontrar este tipo de relaciones. El objetivo de esta comunicación no es ofrecer una detallada revisión bibliográfica sobre los trabajos publicados en este sentido y si el lector tiene interés, dos trabajos interesantes en esta dirección son: Van Dijk et al., 2002 y Angulo-Martínez et al., 2016.

Otro ámbito de estudio importante en el campo de la erosión del suelo es el que utiliza simuladores de lluvia, bien para simular eventos de precipitación sobre distin- 
tos tipos de cultivos o coberturas vegetales; o los estudios centrados en el espectro disdrométrico producido por los aspersores de riego, (Fig. 3).
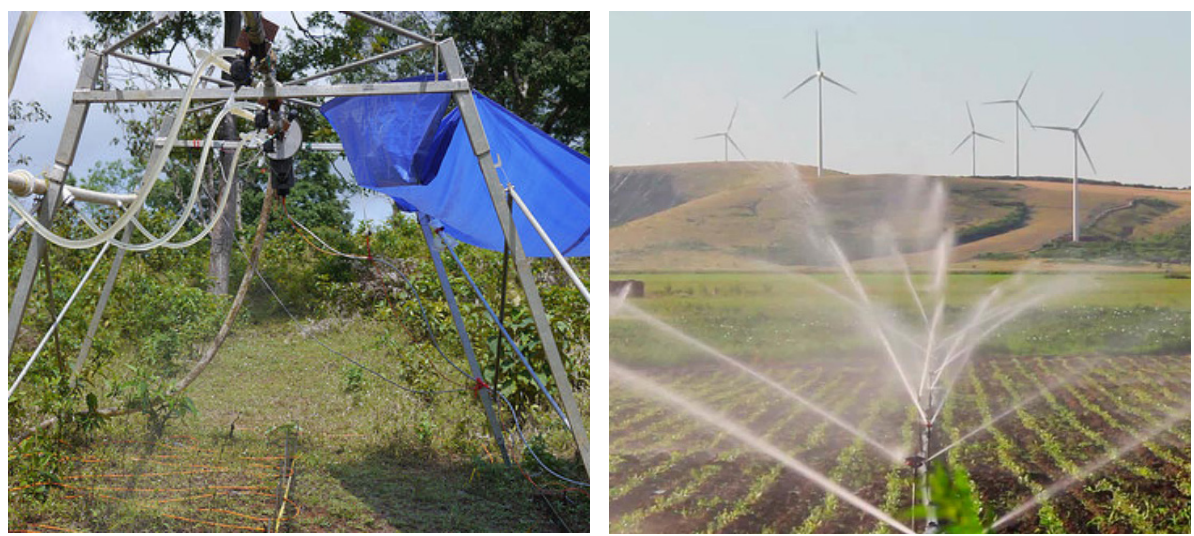

Fig. 3: Fotografía, a la izquierda, de ensayo con simulador de lluvia en un campo de cultivo. Fuente: Center for computational Hydrology and Hydro Sciences. Fotografía a la derecha: riego por aspersión en campo de cultivo.

En el primer caso se trata de investigar como respondería el suelo ante eventos de precipitación, sin embargo el espectro disdrométrico de lluvia simulada dista bastante del de la lluvia real, sobretodo en cuanto a los pulsos de intensidad y las variaciones en los tamaños de gota asociados durante el evento. Las calibraciones, son necesarias y para ello se utilizan en muchos casos disdrómetros (Iserloh, et al., 2013). En el segundo caso, se estudia la distribución de los tamaños de gota, su velocidad, su forma y su ángulo y fuerza de impacto (Bautista-Capetillo, et al., 2014), incluyendo en los objetivos el desarrollo y mejora de sistemas de riego.

El otro gran ámbito de estudio es el de la teledetección de la precipitación. En este se incluyen los estudios tanto de radar como los relacionados la precipitación estimada por satélite y los algoritmos relacionados.

En el ámbito de la precipitación radar, las principales aplicaciones están relacionadas con los estudios de riesgos hidroclimáticos, en especial avenidas e inundaciones, eventos extremos de precipitación y ciclones, entre otras muchas.

Un ejemplo a citar en España, es el Centro de Recerca Aplicada en Hidrometeorología (CRAHI) asociado a la Universidad Politécnica de Cataluña. Cuentan con 4 radares distribuidos por el territorio Catalán y su objetivo es la aplicación de la información radar en hidrología. Sus principales líneas de investigación se centran en:

- El desarrollo de algoritmos y modelos de los procesos que controlan el ciclo del agua superficial.

- El desarrollo de modelos de previsión meteorológica e hidrológica capaces de convertir previsiones de lluvia en previsiones de caudal.

- El desarrollo de técnicas para medir y cuantificar la lluvia, el caudal y otras variables hidrometeorológicas.

- El desarrollo de aplicaciones hidrológicas del radar meteorológico. 
Ofrecen también un curso de postgrado sobre el radar meteorológico y sus aplicaciones en hidrología.

Los últimos años han sido tremendamente prolíficos en cuanto a las investigaciones asociadas al espectro disdrométrico de la precipitación y en especial a la teledetección de la misma. En 1997, la agencia americana NASA, y la japonesa JAXA pusieron en marcha la Mission de Medición de la lluvia tropical (TRMM: Tropical Rainfall Measuring Mission), con el objetivo de investigar la precipitación global entre $\operatorname{los} 35^{\circ} \mathrm{N}$ y los $35^{\circ} \mathrm{S}$ y generar productos que pudieran ser utilizados con fines científicos. La mision puso en órbita un satélite que incluía los siguientes instrumentos: radar meteorológico (PR), generador de imágenes por microondas del (TMI), Escáner del Visible y del Infrarrojo (VIRS), Sistema de Energía Radiante de la Tierra y de las Nubes (CERES), y Sensor de Imágenes de Relámpagos (LIS). A partir de estos sensores generaron una serie de productos, entre los que destaca: la precipitación global corregida cada $3 \mathrm{H} 0.25^{\circ} \times 0.25^{\circ}$ (3B42). Los sensores del satélite se apagaron el 8 de abril de 2015 y éste entró en la Tierra el 15 de Junio de 2015. La misión TRMM finalizó tras 17 años para poner en marcha una nueva_Global Precipitation Measurement Mission (GPM). GPM está constituida por una red internacional de satélites que tiene por objetivo el estudio de la lluvia y la nieve a escala global. El satélite fundamental, lanzado el 27 de febrero de 2014, cuenta con un sistema avanzado de radiómetro y radar, para medir la precipitación desde el espacio y servir como referencia estándar capaz de unificar las diferentes medidas de la constelación de satélites que incluye la misión. Las posibles aplicaciones se engloban en una mejor comprensión del ciclo de agua y energía, y la mejora en las previsiones y proyecciones meteorológicas con el fin de prevenir desastres climáticos y en general aportar información climática de calidad que beneficie a la sociedad.

Las mejoras realizadas en los instrumentos a bordo del satélite principal que compone la GPM, así como en los algoritmos para la obtención y depuración de los datos, han sido posibles gracias a varias campañas de validación en tierra. Algunas de ellas son: LPVEX (2010, Finlandia), MC3E (2011, Oklahoma, US), GPCEX (2012, Canadá) e IFLOODS (2013, Iowa). Las dos últimas campañas (IPHEX, 2014, Carolina del Norte, y OLYMPEX, 2015, en el estado de Washington, US) realizadas por la NASA y universidades e institutos asociados forman parte de la validación de la nueva misión, GPM.

Un ejemplo de las campañas de validación se aprecia en la figura 4. La campaña de validación IFLOODS tenía por objetivo mejorar la predicción de avenidas e inundaciones tras la que sufrió Iowa City en 2008. El periodo de observación intensiva duró desde el 1 de mayo al 15 de junio de 2013. Durante ese tiempo se tomaron medidas con varios sensores, tanto proporcionados por la NASA como otros diseñados por el centro de inundaciones de Iowa (Iowa Flood Center). Estas medidas incluían dos tipos de radar y un transecto de disdrómetros (con 2 tipos de disdrómetros), capaces de validar los hidrometeoros que el radar iba captando.

La campaña fue muy afortunada con la precipitación. Se produjeron avenidas, consecuencia de lluvias intensas, cuya subida de caudal estaba monitorizada a tiempo real por sensores instalados en los principales ríos. Hubo también eventos de preci- 
pitación sólida, nieve y granizo, así como lluvias procedentes de distintos tipos de situaciones atmosféricas.

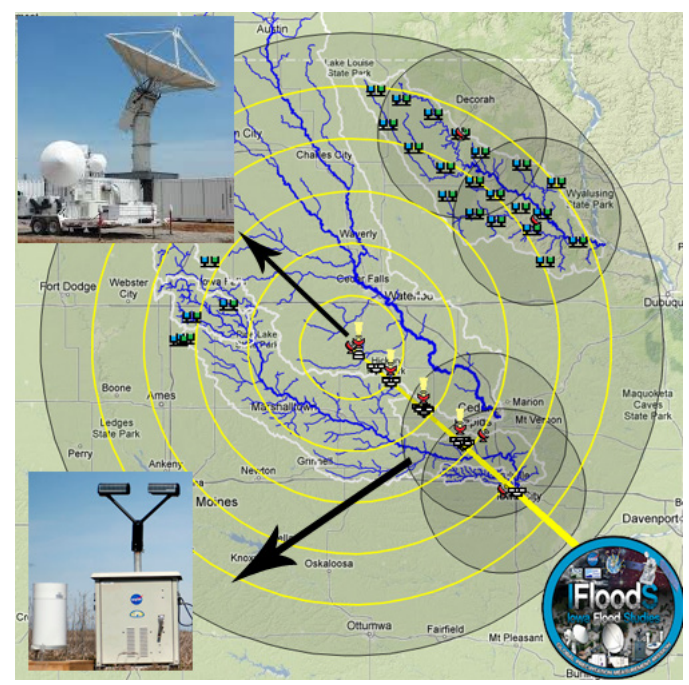

Fig. 4: Emplazamiento de los sensores para la campaña de validación de TRMM, IFLOODS en el estado de Iowa, US. Se marca el transecto de disdrómetro-pluviómetros. Fuente: IFLOODS NASA portal https://pmm.nasa.gov/IFloodS

Los resultados obtenidos en estas campañas, que entre otras cosas, giran en torno al espectro disdrométrico de la precipitación, aportan un volumen de datos fundamental para mejorar la detección, comprensión y modelización de los distintos eventos de precipitación y sobre como afectan la forma de vida de las distintas sociedades.

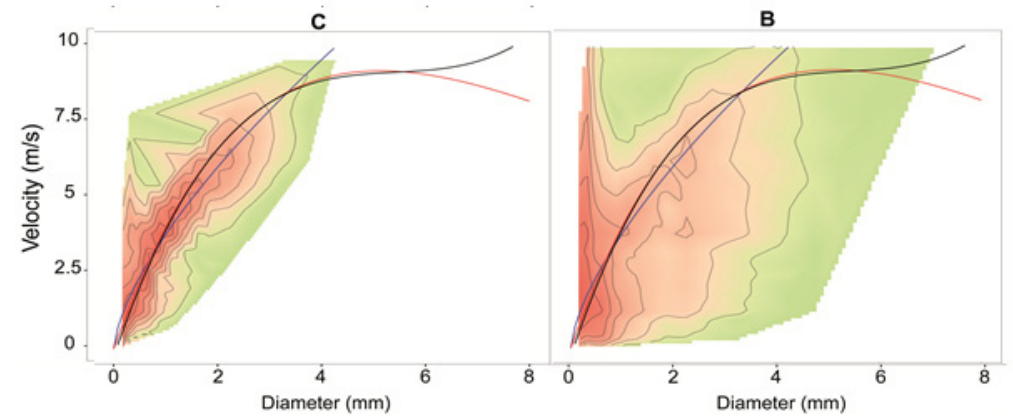

Fig. 5: Relación entre la velocidad de las gotas en función de su tamaño para eventos de baja intensidad (izquierda) y de alta intensidad (derecha). Fuente: elaboración propia, (AnguloMartínez et al., 2016).

A pesar de todo ello, resulta necesario seguir investigando. A pesar de ser la importancia que tiene el objeto de estudio presentado y su transversalidad, unos campos están más desarrollados que otros y no todos los disdrómetros ofrecen medidas sufi- 
cientemente precisas. El efecto del viento y el propio error e incertidumbre asociado al sensor puede impedir en ocasiones llegar a conclusiones claras sobre lo que se observa. A modo de ejemplo se incluye la figura 5, que muestra como la relación entre los tamaños de gota y su velocidad de caída, depende de la intensidad del evento y muestra evidencias de procesos meteorológicos de coalescencia y rotura a lo largo de la columna de lluvia.

\section{CONCLUSIÓN}

La presente comunicación ha tratado de explicar brevemente en qué consiste el espectro disdrométrico de la precipitación, su interés científico y como se han ido desarrollando sensores con el fin de ofrecer una buena determinación del mismo. En esta comunicación se ha tratado de incluir los ámbitos científicos relacionados con el objeto de estudio, y cuáles han sido los principales desarrollos e hitos en los mismos.

\section{AGRADECIMIENTOS}

Este trabajo ha contado con financiación del proyecto DISDROPEC: CGL201124185. El trabajo de M. Angulo-Martínez ha sido posible gracias a un contrato postdoctoral del programa Juan de la Cierva del Ministerio Español de Economía y Competitividad. Esta autora quiere agradecer la oportunidad de haber podido participar en las campañas de validación de la NASA IFLOODs y IPHEX.

\section{REFERENCIAS}

Angulo-Martínez, M., Beguería, S., Kyselý, J., 2016. Use of disdrometer data to evaluate the relationship of rainfall kinetic energy and intensity (KE-I). Science of the Total Environment, 568, 83-94.

Bautista-Capetillo, C., Robles, O., Salinas, H., Playán, E. 2014. A particle tracking velocimetry technique for drop characterization in agricultural sprinklers, Irrigation Science, 32(6), 437-447.

Blanchard, D.C. 1953. Raindrop size-distribution in hawaiian rains. Journal of Meteorology, 10(6), 457-473.

Bentley, W.A. 1904. Studies of raindrops and raindrop phenomena, Monthly Weather Review, 32, 450-456.

Hauser, D., Amayenc, P., Nutten, B., Waldteufel, P., 1984. A new optical instrument for simultaneous measurement of raindrop diameter and fall speed distributions. J. Atmos. Ocean. Technol. 1, 256-269.

Iserloh, T., Ries, J.B., Arnáez, J., Boix-Fayos, C., Butzen, V., Cerdà, A., Echeverría, M.T., Fernández-Gálvez, J., Fister, W., Geißler, C., Gómez, J.A., Gómez-Macpherson, H., Kuhn, N.J., Lázaro, R., León, F.J., Martínez-Mena, M., Martínez-Murillo, J.F., Marzen, M., Mingorance M.D., Ortigosa, L., Peters, P., Regüés, D., Ruiz-Sinoga, J.D., Scholten, T., Seeger, M., Solé-Benet, A., Wengel, R., Wirtz, S. 2013. European small portable rainfall simulators: A comparison of rainfall characteristics. Catena, 110, 100-112.

Joss, J y Waldvogel, A. 1967. Ein Spektrograph für Niederschlagstropfen mit automatischer Auswertung. Pure and Applied geophysics, 68(1), 240-246. 
Kruger, A., Krajewski, W.F. 2002. Two Dimensional Video-Disdrometer: a description. Journal of Atmospheric and Oceanic Technology, 19, 600-617.

Laws, J.O., Parsons, D.A. 1943. The relation of raindrop size to intensity. American Geophysical Union Transactions, 26, 452-460

Lowe, E.J. 1892. Raindrops. Quarterly Journal of the Royal Meteorological Society, 18 (84), 242-245.

Mutchler, C.K., Hansen, L.M. 1970. Splash of a waterdrop at terminal velocity. Transactions of American Geophysical Union, 31, 836-842.

Renard, K.G., Foster, G.R., Weesies, G.A., McCool, D.K., Yoder, D.C., 1997. Predicting Soil Erosion by Water: A Guide to Conservation Planning with the Revised Universal Soil Loss Equation (RUSLE). USDA Agricultural Handbook No. 703. USDA, Washington, DC (384 pp).

Van Dijk, A.I.J.M., Bruijnzeel, L.A., Rosewell, C.J. 2002. Rainfall intensity-kinetic energy relationships: a critical literature appraisal. Journal of Hydrology, 261: $1-23$.

\section{Websites:}

Centro de Recerca Aplicada en Hidrometeorología (CRAHI) http://crahi.upc.edu/

Global Precipitation Measurement Mission (GPM) https://pmm.nasa.gov/GPM

Global Precipitation Measurement Mission - Ground Validation https://pmm.nasa.gov/science/ground-validation 